This is a post-peer-review, pre-copyedit version of an article published in Psychological Trauma: Theory, Research, Practice and Policy. The final authenticated version is available online at:

https://www.ncbi.nlm.nih.gov/pubmed/28682106

Posttraumatic growth in bereaved parents: A multidimensional model of associated factors Sara Albuquerque, Isabel Narciso, and Marco Pereira 


\begin{abstract}
Objective: Although the death of a child is a devastating event, recent evidence shows that personal growth is a relevant outcome of parents' grief. This study aimed to examine the factors associated with posttraumatic growth (PTG) and to propose a multidimensional model consisting of sociodemographic, situational, and intrapersonal and interpersonal factors. Methods: A sample $(N=197 ; 89.8 \%$ female; mean age $=39.44$ years $)$ of bereaved parents completed the Post-Traumatic Growth Inventory-Short Form, the 14-Item Resilience Scale, the Continuing Bonds Scale and the Dyadic Coping Inventory.

Results: The final model consisted of sociodemographic, situational, intrapersonal and interpersonal factors of PTG, which accounted for $36.7 \%$ of the variance. Higher levels of PTG were generally associated with female sex, younger age of the child, higher levels of resilience, higher levels of internalized continuing bonds (i.e., internal representation of the child, maintaining psychological proximity), and higher levels of stress communication by the partner (communicating the stress experience and requesting emotional or practical support).
\end{abstract}

Conclusions: In clinical practice, health professionals assisting bereaved parents should pay attention to men and parents of older children, who might be at higher risk of difficulties in developing PTG. Additionally, promoting a more internalized bond with the child, resilience and dyadic coping, especially stress communication, can constitute important therapeutic goals.

Keywords: death of a child; multidimensional model; parental adjustment; posttraumatic growth. 


\section{Posttraumatic growth in bereaved parents: A multidimensional model of associated}

\section{factors}

Attention to the positive aspects of the aftermath of a trauma has increased over the past years, and several theories and models have focused on systematically defining these aspects (Armeli, Gunthert, \& Cohen, 2001; Calhoun \& Tedeschi, 2001). One positive aspect is posttraumatic growth (PTG), a term proposed by Tedeschi and Calhoun $(1996,2004)$ to refer to the self-reported positive changes that develop beyond one's previous level of psychological functioning as a result of coping with highly challenging life circumstances and their aftermath. According to these authors, PTG is a multidimensional construct that includes changes in the domains of self-perception, interpersonal relationships and philosophy of life.

One assumption in Tedeschi and Calhoun's $(1996,2004)$ model is that for the occurrence of PTG, the event must be sufficiently traumatic, causing extreme distress and challenging one's fundamental understanding of the self, the world and one's place in it. The death of a child qualifies as such because it contests the natural order of life and may shatter parents' basic assumptions of the understanding of the world (Stroebe $\&$ Schut, 2001). The death of a child is also recognized to be among the most challenging and traumatic of losses (Rogers, Floyd, Seltzer, Greenberg, \& Hong, 2008). Bereaved parents report intense grief and high levels of depressive symptoms and anxiety and they have a higher risk of mortality than average in the general population or than their non-bereaved counterparts ( $\mathrm{Li}$, Precht, Mortensen, \& Olsen, 2003; Rogers et al., 2008).

Despite reports showing that parents may be unable to fully recover from the death of their child many years later (Wortman \& Silver, 2001), there is evidence that personal growth is a relevant outcome of parental grief. Specifically, some studies of bereaved parents have described the centrality of PTG (Engelkemeyer \& Marwit, 2008), the perception of increased 
empathy for others, the need to adjust values or reprioritize goals (Miles \& Crandall, 1983) and the experience of competence and strength (Polatinsky \& Esprey, 2000). In this context, an important question that remains is how the experience of PTG can emerge from such intense suffering. The clarification of factors that may influence PTG has been consistently identified as a research priority (Calhoun, Tedeschi, Cann, \& Hanks, 2010). Therefore, the aim of this study was to examine a multidimensional model of factors associated with PTG among bereaved parents. We hypothesized that three main types of factors contribute to PTG after the death of a child: 1) sociodemographic factors (parents and deceased child) and situational factors (factors related to the circumstances of the death); 2) intrapersonal factors, including individual characteristics, such as resilience, and after-death factors, such as continuing bonds i.e., the maintenance of the relationship with the child; and 3) interpersonal factors, such as dyadic coping (DC). The selection of these factors was guided by the existing literature, both in general PTG literature as well as among studies conducted with bereaved parents. Considering the evidence of the negative association between grief and growth (e.g., Engelkemeyer \& Marwit, 2008; Gamino, Sewell, \& Easterling, 2000), Engelkemeyer and Marwit (2008) argued that factors that help in coping with grief may also promote the perception of growth. Therefore, studies that used grief as an outcome were considered in the hypothesis of this study.

\section{Sociodemographic and situational factors}

A review by Linley and Joseph (2004) on positive change following adversity underlined the existence of gender differences in PTG in several contexts (e.g., breast cancer, bereavement, illness or accident). In a sample of men and women who were traumatized by various events, Tedeschi and Calhoun (1996) found that perceptions of growth by traumatized women were double of those by traumatized men, suggesting that women may be more able than men to learn and benefit from difficult life experiences. Other studies in 
various contexts have reported similar results, revealing that higher levels of PTG have been reported among women (Vishnevsky, Cann, Calhoun, Tedeschi, \& Demakis, 2010; Weiss, 2002). Additionally, the PTG model assumption that growth takes time to emerge (Tedeschi \& Calhoun, 1995) has been confirmed in the parental bereavement literature, which indicates that time since death correlates with higher PTG (Engelkemeyer \& Marwit, 2008; Scholtes \& Browne, 2015).

Research with bereaved parents has demonstrated the protective role of having other children. Dyregrov, Nordanger and Dyregrov (2003) found that one of the most significant predictors of psychosocial distress was not having surviving children. Similarly, Wijngaardsde Meij et al. (2005) found that parents had lower levels of grief when they had other children. In this study, the authors also found that higher age of the child was related to higher grief; this finding was also confirmed in a study by Lichtenthal, Currier, Neimeyer, and Keesee (2010). More recently, Scholtes and Browne (2015) found that child's age at death was negatively associated with personal growth. Regarding the factors related to the child's death, the unexpectedness of the death has been found to be associated with poorer parental outcomes (e.g., more intense grief; Barry, Kasl, \& Prigerson, 2002; Wijngaards-de Meij et al., 2005). Another important feature is whether parents, according to their own perception, said goodbye to their child. The general bereavement literature attests that saying goodbye is salutary (Gamino et al., 2000; Schut, de Keijser, van den Bout, \& Dijkhuis, 1991). This finding was supported in a longitudinal study with bereaved parents that found that the feeling of having said goodbye to the child was associated with lower levels of grief (Wijngaards-de Meij et al., 2008).

\section{Intrapersonal factors}

Schaefer and Moos (2001) proposed that personal resources affect individuals' appraisal and coping during and after bereavement, which in turn affect post-bereavement 
outcomes, such as personal growth. One of such personal resources is resilience, with evidence showing it's positive association with growth (Bensimon, 2012) and centrality for PTG during grief (Znoj, Kruit, \& Wuthrich, 2004). Continuing bonds with the child, or the continued connection after death, has been proposed as another relevant factor associated with PTG (Field \& Filanosky, 2010). These authors specifically identified two forms of continuing bonds: internalized continuing bonds, which are defined as expressions involving use of the deceased as an autonomy-promoting secure base, and externalized continuing bonds, which refer to expressions involving illusions and hallucinations of the deceased. Continuing bonds have been identified as an integral component of adaptation to bereavement, and recent works have proposed that the adaptiveness of continuing bonds might depend on the form of their expression for the bereaved (Stroebe \& Schut, 2005). When examining the association between internalized and externalized continuing bonds and PTG, Field and Filanosky (2010) found that internalized continuing bonds were uniquely positively associated with personal growth, a link that has been confirmed in recent studies (Scholtes \& Browne, 2015).

\section{Interpersonal factors}

Most empirical work on PTG has focused on individuals without considering the impact of their social support networks. This lack of a perspective that accommodates interpersonal processes has been criticized by several authors (e.g., Calhoun \& Tedeschi, 2006). A growing body of recent work has suggested that close relationships can support PTG. Relationship quality, self-disclosure, and support seeking were found to be associated with greater PTG (e.g., Hungerbuehler, Vollrath, \& Landolt, 2011; Lindstrom, Cann, Calhoun, \& Tedeschi, 2013; Prati \& Pietrantoni, 2009). Studies have also shown that partner support can serve as a basis for PTG and PTG-related concepts (e.g., meaning finding) in several loss contexts (Canevello, Michels, \& Hilaire, 2016), including parental bereavement 
(Albuquerque, Pereira, \& Narciso, 2016; Polatinsky \& Esprey, 2000). In this context, DC may be considered a key interpersonal factor associated with PTG. Dyadic coping consists of the efforts of one partner to support the other when he or she is stressed and both partners' mutual attempts to cope with a shared stressor (Bodenmann, 2005). Because bereaved parents concurrently experience the traumatic event that is the death of a child, mutual support between individuals who truly understand each other's experience may be particularly important for individual adjustment. Hence, DC may be a potentially sound resource for the development of PTG after the death of a child.

\section{The present study}

The aim of this study was to examine the combined contribution of factors at different levels (sociodemographic, situational, intrapersonal and interpersonal) to PTG among bereaved parents. The findings from this study will add to current knowledge concerning PTG as an outcome of parental bereavement by testing a multidimensional model of factors and focusing on newly or rarely addressed variables (e.g., having said goodbye to the child, continuing bonds, dyadic coping).

\section{Method}

\section{Participants and procedure}

The present study was approved by the Ethics Committees of the Faculty of Psychology and Education Sciences of the hosting institution and several hospitals across the country. Participants were recruited among the general population (through internet dissemination using bereavement associations' mailing lists, bereavement blogs or groups in social networks) and through references from mental health professionals, between November 2013 and May 2015.

Participants completed an online survey on the website of the hosting institution or a paper version of the set of questionnaires. Informed consent was attached to both forms of the 
assessment protocol (online or paper) and included detailed information about the study's aims, the inclusion criteria, the participants' and researchers' roles, the confidentiality and anonymity of the answers and the possible risks associated with participation in the study (e.g., triggering of painful memories and emotions).

The following inclusion criteria were defined: (1) having lost a child (of any age) by death; (2) being married or cohabiting; (3) minimum time since death of six months (to avoid the crisis period; e.g., Stroebe, Stroebe, \& Schut, 2003); (4) being at least 18 years of age; and (5) having sufficient knowledge of language to complete the set of questionnaires. In the paper version, participants were asked to complete the assessment protocol and to return it anonymously in a sealed envelope either to the health professionals or directly to the researcher (first author).

The final sample consisted of 197 parents with a mean age of 39.44 years (standard deviation $[S D]=11.32$; range: $18-79)$. The parents were mostly female $(89.8 \%)$, and married $(86.3 \%)$ or cohabiting $(13.7 \%)$. The length of the marital relationship ranged between 0 and 56 years $(M=16.70 ; S D=11.95)$. Years of education ranged from 3 to 19 years $(M=13.41$; $S D=3.89)$. Most parents were employed $(59.9 \%)$ and had other children $(71.1 \%)$. The deceased offspring were mostly male (59.4\%). The age of the deceased offspring ranged from stillborn to 52 years $(M=8.96 ; S D=12.35)$, and the time since death ranged from six months to 10 years $(M=2.76$ years; $S D=2.34)$. The causes of death included fetal death $(27.4 \%)$, illness (23.4\%), accident (16.2\%), neonatal death (16.2\%), sudden death (7.6\%), suicide $(4.6 \%)$ or homicide $(4.1 \%)$. For most parents $(81.2 \%)$, the death of the child was unexpected.

\section{Measures}

Sociodemographic and situational factors. A self-reported questionnaire, purposely developed for this study, was used to collect parents' and offspring's sociodemographic data and information regarding the circumstances of the child's death. Parents provided 
information on sex, age, marital status (single, married, cohabiting, separated/divorced or widowed), length of the marital relationship, years of education, employment status (employed, unemployed, retired or student), existence of other children (yes/no question), deceased child's age and sex, time since death, cause of death (fetal death, illness, accident, neonatal death, sudden death, suicide or homicide), expectedness of death (yes/no question), place of death (hospital, home or other), and having said goodbye (yes/no question).

Post-Traumatic Growth Inventory-Short Form (PTGI-SF). The PTGI-SF (Cann et al., 2010) assesses perceived positive outcomes of traumatic or stressful events on the following five subscales: personal strength, spiritual change, relating to others, appreciation of life, and new possibilities. The PTGI-SF comprises 10 items with six response alternatives, ranging from 0 (no change) to 5 (high degree of change). Scores on the PTGI-SF range between 0 and 50, with higher scores reflecting a higher level of PTG. The original study of the PTGI-SF, which included a sample of bereaved parents, showed satisfactory reliability and validity (Cann et al., 2010), which was later replicated in studies with other samples (Kaler, Erbes, Tedeschi, Arbisi, \& Polusny, 2011; Lamela, Figueiredo, Bastos, \& Martins, 2014). In this study, the total of the scale was used (Cronbach's alpha $=.90$ ).

14-Item Resilience Scale (RS-14). The RS-14 (Wagnild \& Young, 1993) is a 14-item scale that assesses resilience, that is, the capacity to endure life stressors and to thrive and make meaning from challenges. Respondents are asked to rate the items on a seven-point response scale ranging from 1 (strongly disagree) to 7 (strongly agree). Scores on the RS-14 range between 14 and 70. Higher scores denote higher resilience. The RS-14 has been shown to be reliable and valid across multiple studies (Nishi et al., 2013; Oliveira, Matos, Pinheiro, \& Oliveira, 2015). In the current study, the Cronbach's alpha was .92.

Continuing Bonds Scale (CBS-16). The CBS-16 (Field \& Filanosky, 2010) assesses the maintenance of the relationship with the lost child. This scale is organized into two 
subscales: internalized continuing bonds (internalized CB; 10 items) and externalized continuing bonds (externalized CB; 6 items). The first subscale refers to an abstract internal representation of the deceased, or maintaining psychological proximity. The second consists of the search for physical proximity and can be expressed by delusions and hallucinations. The items are answered on a four-point response scale ranging from 0 (Never) to 3 (Very often), referring to experiences in the last month. Scores on the CBS-16 range between 0 and 32, with higher scores reflecting a higher level of continuing bonds. The good reliability and validity of CBS-16 has been demonstrated previously (De Luca et al., 2016). In this study, internal consistency was .86 for internalized $\mathrm{CB}$ and .85 for externalized CB.

Dyadic Coping Inventory (DCI). The DCI (Bodenmann, 2008) is a 37-item inventory that assesses stress communication and DC as perceived by each partner about their own coping, each partner's perception of the other's coping and each partner's view of how they cope as a couple. The DCI is organized into seven subscales: stress communication (by oneself and by the partner) includes the ability to communicate the stress experience and to request emotional or practical support; positive DC (by oneself and by the partner) includes supportive behaviors (one partner expressing understanding and solidarity or providing information and practical advice to the other) and delegated behaviors (one partner taking over certain tasks and responsibilities to relieve the partner's burden following an explicit request from the other partner); negative DC (by oneself and by the partner) includes hostility toward the partner; and joint DC refers to mutual attempts by both partners to cope with a shared stressor together and involves joint problem solving and information seeking, shared feelings, mutual commitment, or joint relaxation (Bodenmann, 2005). The items are answered on a five-point scale ranging from 1 (Very rarely) to 5 (Very often). The mean of the respective items serves as the subscales' total scores. Higher scores on the positive and joint DC subscales and lower scores on the negative DC subscales indicate better DC. 
Adequate reliability and validity for the DCI was demonstrated in numerous studies (Ledermann et al., 2010; Vedes, Nussbeck, Bodenmann, Lind, \& Ferreira, 2013). In this study, all Cronbach's alphas were above .70, with the exception of the negative DC by oneself subscale $(\alpha=.67)$.

\section{Data analysis}

Data analyses were conducted using the Statistical Package for the Social Sciences (IBM SPSS, version 20.0). Descriptive statistics were calculated to explore the sample's characteristics. Pearson's correlations were performed to assess the associations between the study variables. Hierarchical Multiple Regression (HRM) analysis was conducted to examine the factors associated with PTG. Prior to conducing HRM, the relevant assumptions were tested (sample size, collinearity statistics) and the categorical variables (parents' gender, having other children, unexpectedness of death and having said goodbye) were dummy coded. Research suggests that the observations of the two members of a given couple cannot be treated as independent observations (Kenny, Kashy, \& Cook, 2006). In this study, when complete couples were included as separate observations in the individual-level data file, one member of the couple was randomly excluded. According to Cohen's (1992) guidelines, $f^{2} \geq$ $0.02, f^{2} \geq 0.15$, and $f^{2} \geq 0.35$ represent small, medium, and large effect sizes, respectively.

\section{Results}

\section{Preliminary analysis}

Table 1 presents the descriptive statistics and the Pearson correlations between the study variables. All variables were significantly correlated with PTG, except for parents' gender, having other children, unexpectedness of death, time since death, externalized bonds, and negative DC by oneself and by the partner. Child's age was negatively correlated with PTG. Having said goodbye to the child, resilience, internalized CB and the remaining DC forms were positively correlated with PTG. 


\section{Hierarchical multiple regressions}

To examine the factors associated with PTG, HRM were conducted. Given the high correlation between joint DC and other DC forms, particularly positive DC by the partner ( $r$ $=.78, p<.001)$, joint DC was not included in the regression model. The regression models consisted of three steps. Step 1 included the sociodemographic (parents' gender, having other children and child's age) and situational factors (unexpectedness of death, having said goodbye and time since death); intrapersonal factors (resilience and internalized and externalized CB) were entered in step 2; and the interpersonal factors (forms of DC) was added in step 3. The collinearity statistics (Variance Inflation Factor [VIF] < 10; tolerance > 0.2; Condition Index < 30) suggested that there were no multicollinearity problems.

$$
\text { [INSERT TABLE } 2 \text { HERE] }
$$

\section{[INSERT TABLE 3 HERE]}

The final models are displayed in Table 2. In step 1, parents' gender (being female) and the possibility of saying goodbye were significantly associated with increased PTG, explaining $10.3 \%$ of the variance in PTG. When the intrapersonal factors were added (step 2), resilience and internalized CB were significantly associated with increased PTG and accounted for $20.7 \%$ of the additional variance in PTG. This addition was significant, and the effect size attributable to the addition of intrapersonal factors was medium to large [Cohen's $\left.f^{2}=0.30\right]$. Finally, in step 3 , when adding the forms of DC, only stress communication by the partner was significantly associated with PTG, and explained $5.7 \%$ of the additional variance [Cohen's $\left.f^{2}=0.09\right]$. The final model accounted for $36.7 \%$ of the total variance.

\section{Discussion}

This study examined the association between PTG and sociodemographic and situational factors as well as intra- and interpersonal variables among bereaved parents. The 
main findings indicate that sociodemographic and situational factors and, in particular, intrapersonal factors were significantly associated with PTG. Specifically, higher levels of PTG were significantly associated with female sex, younger age of the child, higher levels of resilience and of internalized continuing bonds, and higher levels of stress communication by the partner.

In this study, women reported higher levels of PTG. This result has been consistently reported in the literature (for a review, see Linley \& Joseph, 2004; Vishnevsky et al., 2010) and supports the suggestion of Tedeschi and Calhoun (1996) that women are more likely than men to derive benefits from challenging life events. Some potential underlying processes may lead to overall gender differences in PTG. One refers to the women's tendency for engaging in more deliberate rumination than men (Treynor, Gonzalez, \& Nolen-Hoeksema, 2003), possibly about aspects such as increased awareness of personal strengths or an appreciation of the importance of social connections, which have been suggested to be associated with higher levels of PTG (Tedeschi \& Calhoun, 2004). An alternate explanation may be the fact that women are more likely to use emotion-focused coping strategies (de Ridder, 2000), which have also been found to be positively associated with PTG (Helgeson, Reynolds, \& Tomich, 2006).

Parents who lost younger children also reported higher levels of PTG, corroborating previous findings of a negative association between child's age and personal growth (Scholtes \& Browne, 2015) and suggesting that there seems to be additional complexities when an older child dies (Wijngaards-de Meij et al., 2005). Authors have suggested that the complexities of a child's death depend on the extensive emotional investment that is made in the relationship with the child (Klass, 1993). Therefore, the attachment bonds may be stronger for parents of older children in comparison with infant deaths (Rogers et al., 2008). Because $27.4 \%$ of the parents in our study lost their child before birth, it is possible that this 
result may be due to the different timings of child's death (before and after birth) in the present sample.

The addition of intrapersonal factors accounted for a significant degree of variance in PTG. Particularly, resilience and internalized CB appeared to be the most relevant factors associated with PTG. The positive association between resilience and PTG is not surprising, and has been previously shown by the positive influence of personal resources and characteristics in coping and appraisals during and after bereavement (Schaefer \& Moos, 2001). More recently, reinforcing this positive association, one study found that stress appraisal as a challenge (positive appraisal) significantly mediated the association between resilience and PTG and was conducive to growth after trauma (Ogińska-Bulik \& Kobylarczyk, 2016). Overall, in the face of adversity, such as when a child dies, resilience, defined as a positive personality characteristic, may enhance the parents' ability for dealing with child's death and, therefore, to promote their growth with less psychological wounds.

The association between internalized CB and PTG also confirms previous findings showing that this form of $\mathrm{CB}$ serves as an important resource in facilitating personal growth (Field \& Filanosky, 2010; Scholtes \& Browne, 2015). The acknowledgement of the reality of death and the use of the child as an internalized secure base in this form of CB may explain its facilitating role in PTG and, therefore, in a more adaptive adjustment to the loss (Field \& Filanosky, 2010). Moreover, as suggested by Scholtes and Browne (2015), internalized CB do not interfere as much as externalized CB with bereaved parents' daily life or relationships, which may explain why internalized CB may have a more relevant role in the development of positive grief outcomes for bereaved parents, such as PTG.

Based on the findings of this study, PTG also seems to be a dyadic process, as shown by the significant associations between PTG and the forms of DC. These associations confirm previous research showing the role of marital support in the promotion of PTG (Canevello et 
al., 2016). However, in the final regression model, only stress communication by the partner (one's perception of the ability of the partner to communicate the stress experience and to request emotional or practical support) was significantly associated with increased PTG. This finding is in agreement with past research showing an association between disclosure about highly stressful events and growth (Taku, Tedeschi, Cann, \& Calhoun, 2009). Indeed, it has been shown that self-disclosure and open communication between bereaved couples may enhance feelings of closeness and understanding (Albuquerque et al., 2016), which in turn may positively influence both partners, namely promoting growth. Our sample is mostly constituted by women, which have been found to value open communication significantly more than men (Kamm \& Vandenberg, 2001). Therefore, it is possible that women may value their partner's openness and good communication (who, eventually, may have less difficulties of sharing his own grief and to be appropriately supportive), thus contributing to a joint grieving process and, consequently, to their PTG. Nevertheless, because this was the first study examining the association between DC forms and PTG, additional research is needed to clarify the associations reported herein. In future studies it would be also valuable to incorporate and expand other interpersonal variables (e.g., dyadic adjustment) in models attempting to explain PTG, to determine whether our results are replicated.

Some non-significant findings were particularly unexpected. Although growth may indeed take time to emerge (Tedeschi \& Calhoun, 1995), in our study, time since the death was not significantly associated with PTG. One might expect increased PTG with longer time since death, only if it allows more time for cognitive processing. Thus, it is possible that the interaction of this variable with time since death may be more important for the occurrence of PTG (Tedeschi \& Calhoun, 2004) than the passage of time alone. Because the death of a child is a unique type of loss, further studies are needed to confirm this hypothesis. The nonsignificant association between unexpectedness of death and PTG is contrary to the evidence 
showing an association between the unexpectedness of death (and feelings of lack of preparedness for it) and more intense grief (e.g., Barry et al., 2002; Wijngaards-de Meij et al., 2005). However, it is in line with one study that did not find differences between survivors of sudden and anticipated death (Carr, House, Wortman, Nesse, \& Kessler, 2001) and that suggested a complex association between bereavement and circumstances of death. To better understand these inconsistencies, future studies with bereaved parents examining the association between preparedness for death and PTG would be of value.

Although having surviving children has been generally associated with more adaptive adjustment and can provide a continued sense of purpose in life (e.g., Wijngaards-de Meij et al., 2005), in this study, having other children and PTG were not associated. However, it has also been suggested that having to take care of other children can be experienced as a burden (Harper, O'Connor, Dickson, \& Ronan, 2011). Accordingly, it is possible that parents may not have the time or energy required to work through their own grief, therefore lessening their possibilities of PTG. Finally, the non-confirmation of the protective role of saying goodbye in the final model was surprising, particularly given the literature that supported the associations and contribution of this variable to PTG (e.g., Engelkemeyer \& Marwit, 2008; Schut et al., 1991). Nevertheless, it is noteworthy that in the model without the forms of DC, parents who perceived having had the possibility of saying goodbye reported higher levels of PTG. To some extent, this association confirms the protective role of saying goodbye that is advocated in bereavement research (Gamino et al., 2000; Schut et al., 1991).

\section{Limitations and future research}

This study is not without limitations. Although we found significant associations between bereaved parents' PTG and sociodemographic, situational, and intra- and interpersonal factors, the study's cross-sectional nature precludes any conclusions regarding causal relationships between the study variables. To overcome this limitation, longitudinal 
studies should be undertaken to determine the directions of the associations reported herein. This is of utmost importance, especially given the risks of therapeutic rejection by bereaved parents if PTG is emphasized too prematurely (Sheikh, 2008). Our findings should also be interpreted with caution in light of the (though common in the bereavement literature; Albuquerque et al., 2016) significant imbalance between men and women, the predominance of Caucasian parents, as well as the heterogeneity of the sample regarding, for instance, the child's age and the causes of death. In future studies, it may be relevant to examine whether the proposed variables' contributions differ depending on these variables, perhaps through a moderation analysis with such variables as moderators. Because poverty has been considered a major stressor that affects bereavement outcomes (Stroebe, Schut, \& Stroebe, 2007), the inclusion of measures of income and socioeconomic status would have been valuable. As well, given the importance of cultural aspects in parents' grieving process (Rosenblatt, 2013) and PTG (Splevins, Cohen, Bowley, \& Joseph, 2010), information on parents' race/ethnicity should also be considered in future research.

\section{Strengths and clinical implications}

This study offers an important contribution to the literature by highlighting alternative pathways that are possible and observable among bereaved parents, such as the possibility of positive outcomes (PTG), thereby broadening the traditional focus on negative outcomes. This study attests to the complexity of PTG by incorporating different and wide-ranging types of factors. This study also demonstrates the complexity within the factors by testing the associations with both forms of CB (internalized and externalized), contrary to general research on continuing bonds that focus solely on CB as a whole (Field \& Filanosky, 2010). Finally, our findings expand current knowledge on the PTG of bereaved parents by focusing on newly or incipiently addressed factors, such as continuing bonds and dyadic coping. Nevertheless, other intrapersonal variables, such as personality characteristics, and 
interpersonal variables, such as marital intimacy, satisfaction with the relationship and partner's support, would also be relevant points of investigation.

This study has some important implications for clinical practice. First, it allows us to identify bereaved parents who may be at higher risk of lower PTG. For example, fathers and parents who have lost older children may require additional clinical attention. Our findings also offer some insight into areas that might prove fruitful in a clinical setting. First, promoting a more internalized nature of the bond to the deceased child and promoting psychological proximity rather than physical proximity (which characterizes externalized continuing bonds) may be important goals in intervention (Field \& Filanosky (2010). Because of the relevant contribution of the perception of saying goodbye, it seems that this variable makes a difference to parents' adjustment. Encouraging parents who did not have the opportunity to say goodbye to find an individualized, symbolic way to say goodbye can constitute an important therapeutic goal (Wijngaards-de Meij, 2008). In addition, parents should be encouraged to make an effort to support each other and, especially, to be open in their communication, by providing information on their grieving experience, as well as how and when they need support from the partner.

Finally, it is important to promote PTG sensitively and with caution. Clinicians should be made aware that promoting PTG should not preclude intervention in coping with intense negative emotions. As several authors have proposed, PTG requires individuals to experience the ability to effectively cope, manage emotions, and experience psychological relief (Calhoun \& Tedeschi, 2001; Tedeschi \& Calhoun, 2004). Moreover, as noted by these authors, parents' difficulty and reluctance to find something good in their loss should be validated, and professionals who assist bereaved parents should not rigidly expect PTG to be an outcome of the intervention. 


\section{References}

Albuquerque, S., Pereira, M., \& Narciso, I. (2016). Couple's relationship after the death of a child: A systematic review. Journal of Child and Family Studies, 25, 30-53. doi:10.1007/s10826-015-0219-2

Armeli, S., Gunthert, K. C., \& Cohen, L. H. (2001). Stressors appraisals, coping and postevents outcomes. Journal of Social and Clinical Psychology, 20, 366-395. doi:10.1521/jscp.20.3.366.22304

Barry L. C., Kasl, S. V., \& Prigerson, H. G. (2002). Psychiatric disorders among bereaved persons: The role of perceived circumstances of death and preparedness for death. American Journal of Geriatric Psychiatry, 10, 447-457. doi:10.1176/appi.ajgp.10.4.447

Bensimon, M. (2012). Elaboration on the association between trauma, PTSD and posttraumatic growth: The role of trait resilience. Personality and Individual Differences, 52, 782-787. doi:10.1016/j.paid.2012.01.011

Bodenmann, G. (2005). Dyadic coping and its significance for marital functioning. In T. Revenson, K. Kayser, \& G. Bodenmann (Eds.), Couples coping with stress: Emerging perspectives on dyadic coping (pp. 33-50). Washington, DC: American Psychological Association.

Bodenmann, G. (2008). Dyadisches Coping Inventar (DCI). Testmanual [Dyadic Coping Inventory (DCI). Test manual]. Bern: Huber.

Calhoun, F. G., \& Tedeschi, R. G. (2001). Posttraumatic growth: The positive lessons of loss. In R. A. Neimeyer (Ed.), Meaning reconstruction and the experience of loss (pp. 157172). Washington, DC: American Psychological Association.

Calhoun, L.G., \& Tedeschi, R.G. (Eds.). (2006). Handbook of posttraumatic growth: Research and practice. Mahwah, NJ: Lawrence Erlbaum Associates. 
Calhoun, L. G., Tedeschi, R. G., Cann, A., \& Hanks, E. A. (2010). Positive outcomes following bereavement: Paths to posttraumatic growth. Psychologica Belgica, 50, 125-143. doi:10.5334/pb-50-1-2-125

Canevello, A., Michels, V., \& Hilaire, N. (2016). Supporting close others' growth after trauma: The role of responsiveness in romantic partners' mutual posttraumatic growth. Psychological Trauma: Theory, Research, Practice, and Policy, 8, 334-342. doi:10.1037/tra0000084

Cann, A., Calhoun, L. G., Tedeschi, R. G., Taku, K., Vishnevsky, T., Triplett, K. N., \& Danhauer, S. C. (2010). A short form of the Posttraumatic Growth Inventory. Anxiety, Stress \& Coping, 23, 127-137. doi:10.1080/10615800903094273

Carr, D., House, J. S., Wortman, C., Nesse, R., \& Kessler, R. C. (2001). Psychological adjustment to sudden and anticipated spousal loss among older widowed persons. The Journals of Gerontology Series B: Psychological Sciences and Social Sciences, 56, 237-248. doi:10.1093/geronb/56.4.s237

Cohen, J. (1992). A power primer. Psychological Bulletin, 112, 155-159. doi:10.1037/00332909.112.1.155

de Ridder, D. (2000). Gender, stress and coping: Do women handle stressful situations differently from men? In L. Sherr \& J. St. Lawrence (Eds.), Women, health and the mind (pp. 115-135). Chichester, UK: Wiley.

De Luca, M. L., Grossi, G., Zaccarello, G., Greco, R., Tineri, M., Slavic, E., ... Palummieri, A. (2016). Adaptation and validation of the "Continuing Bond Scale" in an Italian context. An instrument for studying the persistence of the bond with the deceased in normal and abnormal grief. International Journal of Psychoanalysis and Education, 2, 37-52. 
Dyregrov, K., Nordanger, D., \& Dyregrov, A. (2003). Predictors of psychosocial distress after suicide, SIDS, and accidents. Death Studies, 27, 143-165. doi:10.1080/07481180302892

Engelkemeyer, S. M., \& Marwit, S. J. (2008). Posttraumatic growth in bereaved parents. Journal of Traumatic Stress, 21, 344-346. doi:10.1002/jts.20338

Field, N., \& Filanosky, C. (2010). Continuing bonds, risk factors for complicated grief, and adjustment to bereavement. Death Studies, 34, 1-29. doi:10.1080/07481180903372269

Gamino, L. A., Sewell, K. W., \& Easterling, L. W. (2000). Scott and White grief study-Phase 2: Toward an adaptive model of grief. Death Studies, 24, 633-660. doi:10.1080/07481180050132820

Harper, M., O'Connor, R., Dickson, A., \& Ronan, O. C. (2011). Mothers continuing bonds and ambivalence to personal mortality after the death of their child: An interpretative phenomenological analysis. Psychology, Health \& Medicine, 16, 203-214. doi:10.1080/13548506.2010.532558

Helgeson, V. S., Reynolds, K. A., \& Tomich, P. L. (2006). A meta-analytic review of benefit finding and growth. Journal of Consulting and Clinical Psychology, 74, 797-815. doi:10.1037/0022-006X.74.5.797

Hungerbuehler, I., Vollrath, M. E., \& Landolt, M. A. (2011). Posttraumatic growth in mothers and fathers of children with severe illnesses. Journal of Health Psychology, 16, 1259-1267. doi:10.1177/1359105311405872

Kaler, M. E., Erbes, C. R., Tedeschi, R. G., Arbisi, P. A., \& Polusny, M. A. (2011). Factor structure and concurrent validity of the Posttraumatic Growth Inventory-Short Form among veterans from the Iraq War. Journal of Traumatic Stress, 24, 200-207. doi:10.1002/jts.20623 
Kamm, S., \& Vandenberg, B. (2001). Grief communication, grief reactions and marital satisfaction in bereaved parents. Death Studies, 25, 569-582. doi:10.1080/07481180126576

Kenny, D. A., Kashy, D. A., \& Cook, W. L. (2006). Dyadic data analysis. New York, NY: Guilford Press.

Klass, D. (1993). Solace and immortality: Bereaved parents' continuing bond with their children. Death Studies, 17, 343-368. doi:10.1080/07481189308252630

Lamela, D., Figueiredo, B., Bastos, A., \& Martins, H. (2014). Psychometric properties of the Portuguese version of the Posttraumatic Growth Inventory Short Form among divorced adults. European Journal of Psychological Assessment, 30, 3-14. doi:10.1027/1015-5759/a000161

Ledermann, T., Bodenmann, G., Gagliardi, S., Charvoz, L., Verardi, S., Rossier, J., ... Iafrate, R. (2010). Psychometrics of the Dyadic Coping Inventory in three language groups. Swiss Journal of Psychology, 69, 201-212. doi:10.1024/1421-0185/a000024

Li, J., Precht, D. H., Mortensen, P. B., \& Olsen, J. (2003). Mortality in parents after death of a child in Denmark: A nationwide follow-up study. Lancet, 36, 363-367. doi:10.1016/s0140-6736(03)12387-2

Lichtenthal, W. G., Currier, J. M., Neimeyer, R. A., \& Keesee, N. J. (2010). Sense and significance: A mixed methods examination of meaning making after the loss of one's child. Journal of Clinical Psychology, 66, 791-812. doi:10.1002/jclp.20700

Lindstrom, C. M., Cann, A., Calhoun, L. G., \& Tedeschi, R. G. (2013). The relationship of core belief challenge, rumination, disclosure, and sociocultural elements to posttraumatic growth. Psychological Trauma, 5, 50-55. doi:10.1037/a0022030

Linley, P. A., \& Joseph, S. (2004). Positive change following trauma and adversity: A review. Journal of Traumatic Stress, 17, 11-21. doi:10.1023/b:jots.0000014671.27856.7e 
Miles, M. S., \& Crandall, E. K. (1983). The search for meaning and its potential for affecting growth in bereaved parents. Health Values: Achieving High Level Wellness, 7, 19-23. doi:10.1007/978-1-4684-7021-5_17

Nishi, D., Uehara, R., Yoshikawa, E., Sato, G., Ito, M., \& Matsuoka, Y. (2013). Culturally sensitive and universal measure of resilience for Japanese populations: Tachikawa Resilience Scale in comparison with Resilience Scale 14-item version. Psychiatry and Clinical Neurosciences, 67, 174-181. doi:10.1111/pcn.12028

Ogińska-Bulik, N., \& Kobylarczyk, M. (2016). Association between resiliency and posttraumatic growth in firefighters: The role of stress appraisal. International Journal of Occupational Safety and Ergonomics, 22, 40-48.

doi:10.1080/10803548.2015.1109372

Oliveira, A., Matos, A. P., Pinheiro, M. R., \& Oliveira, S. (2015). Confirmatory factor analysis of the resilience scale short form in a Portuguese adolescent sample. Procedia-Social and Behavioral Sciences, 165, 260-266. doi:10.1016/j.sbspro.2014.12.630

Polatinsky, S., \& Esprey, Y. (2000). An assessment of gender differences in the perception of benefit resulting from the loss of a child. Journal of Traumatic Stress, 13, 709-718. doi:10.1023/A:1007870419116

Prati, G., \& Pietrantoni, L. (2009). Optimism, social support, and coping strategies as factors contributing to posttraumatic growth: A meta-analysis. Journal of Loss and Trauma, 14, 364-388. doi:10.1080/15325020902724271

Rogers, C. H. (2005). The effect of the death of a child on midlife mental and physical health: An exploration of risk and resilience factors. Unpublished doctoral thesis, Georgia State University, Atlanta. 
Rosenblatt, P. C. (2013). Family grief in cross-cultural perspective. Family Science, 4, 12-19. doi:10.1080/19424620.2013.819226

Schaefer, J. A., \& Moos, R. H. (2001). Bereavement experiences and personal growth. In M. S. Stroebe, R. O. Hansson, W. Stroebe, \& H. Schut (Eds.), Handbook of bereavement research: Consequences, coping, and care (pp. 145-167). Washington, DC: American Psychological Association.

Scholtes, D., \& Browne, M. (2015). Internalized and externalized continuing bonds in bereaved parents: Their relationship with grief intensity and personal growth. Death Studies, 39, 75-83. doi:10.1080/07481187.2014.890680

Schut, H. A. W., de Keijser, J., Van den Bout, J., \& Dijkhuis, J. H. (1991). Post-traumatic stress symptoms in the first year of conjugal bereavement. Anxiety Research, 4, 225234. doi:10.1080/08917779108248776

Sheikh, A. I. (2008). Posttraumatic growth in trauma survivors: Implications for practice, Counselling Psychology Quarterly, 21, 85-97. doi:10.1080/09515070801896186

Splevins, K., Cohen, K., Bowley, J., \& Joseph, S. (2010). Theories of posttraumatic growth: Cross-cultural perspectives. Journal of Loss and Trauma, 15, 259-277. doi:10.1080/15325020903382111

Stroebe, M. S., \& Schut, H. (2001). Meaning making in the Dual Process Model of Coping with Bereavement. In R. A. Neimeyer (Ed.), Meaning reconstruction and the experience of loss (pp. 55-73). Washington, DC: American Psychological Association.

Stroebe, M., \& Schut, H. (2005). To continue or relinquish bonds? A review of consequences for the bereaved. Death Studies, 29, 477-494. doi:10.1080/07481180590962659

Stroebe, M., Schut, H., \& Stroebe, W. (2007). Health outcomes of bereavement. Lancet, 370, 1960-1973. doi:10.1016/S0140-6736(07)61816-9 
Stroebe, M., Stroebe, W., \& Schut, H. (2003). Bereavement research: Methodological issues and ethical concerns. Palliative Medicine, 17, 235-240. doi:10.1191/0269216303pm768rr

Rogers, C. H., Floyd, F. J., Seltzer, M. M., Greenberg, J., \& Hong, J. (2008). Long-term effects of the death of a child on parents' adjustment in midlife. Journal of Family Psychology, 22, 203-211. doi:10.1037/0893-3200.22.2.203.

Taku, K., Tedeschi, R. G., Cann, A., \& Calhoun, L. G. (2009). The culture of disclosure: Effects of perceived reactions to disclosure on posttraumatic growth and distress in Japan. Journal of Social and Clinical Psychology, 28, 1226-1243. doi:10.1521/jscp.2009.28.10.1226

Tedeschi, R. G., \& Calhoun, L. G. (1995). Trauma and transformation: Growing in the aftermath of suffering. Thousand Oaks, CA: Sage.

Tedeschi, R. G., \& Calhoun, L. G. (1996). The posttraumatic growth inventory: Measuring the positive legacy of trauma. Journal of Traumatic Stress, 9, 455-471. doi:10.1007/bf02103658

Tedeschi, R. G., \& Calhoun, L. G. (2004). Posttraumatic growth: Conceptual foundations and empirical evidence. Psychological Inquiry, 15, 37-41. doi:10.1207/s15327965pli1501

Treynor, W., Gonzalez, R., \& Nolen-Hoeksema, S. (2003). Rumination reconsidered: A psychometric analysis. Cognitive Therapy and Research, 27, 247-259. doi:10.1023/A:1023910315561

Vedes, A., Nussbeck, F. W., Bodenmann, G., Lind, W., \& Ferreira, A. (2013). Psychometric properties and validity of the Dyadic Coping Inventory in Portuguese. Swiss Journal of Psychology, 72, 149-157. doi:10.1024/1421-0185/a000108 
Vishnevsky, T., Cann, A., Calhoun, L. G., Tedeschi, R. G., \& Demakis, G. J. (2010). Gender differences in self-reported posttraumatic growth: A meta-analysis. Psychology of Women Quarterly, 34, 110-120. doi:10.1111/j.1471-6402.2009.01546.x

Wagnild, G. \& Young, H. (1993). Development and psychometric evaluation of the resilience scale. Journal of Nursing Measurement, 1, 165-177. doi:10.3724/sp.j.1041.2008.00902

Weiss, T. (2002). Posttraumatic growth in women with breast cancer and their husbands: An intersubjective validation study. Journal of Psychosocial Oncology, 20, 65-80. doi:10.1300/j077v20n02_04

Wijngaards-de Meij, L., Stroebe, M., Schut, H., Stroebe, W., van den Bout, J., van der Heijden, P., \& Dijkstra, I. (2005). Couples at risk following the death of their child: Predictors of grief versus depression. Journal of Consulting and Clinical Psychology, 73, 617-623. doi:10.1037/0022-006X.73.4.617

Wijngaards-de Mej, L., Stroebe, M., Stroebe, W., Schut, H., van den Bout, J., van der Heijden, P. G. M., \& Dijkstra, I. (2008). The impact of circumstances surrounding the death of a child on parents' grief. Death Studies, 32, 237-252. doi:10.1080/07481180701881263

Wortman, C., \& Silver, R. (2001). The myths of coping with loss revisited. In M. Stroebe, R. O. Hansson, W. Stroebe, \& H. A. W. Schut (Eds.), Handbook of bereavement research: Consequences, coping and care (pp. 405-429). Washington DC: American Psychological Association.

Znoj, H. J., Kruit, \& Wuthrich, C. (2004). The resource model of grief. A structural equation modeling approach. Unpublished manuscript, University of Bern. 
POSTTRAUMATIC GROWTH IN BEREAVED PARENTS 27

Table 1. Descriptive statistics and Pearson's correlations for the study variables

\begin{tabular}{|c|c|c|c|c|c|c|c|c|c|c|c|c|c|c|c|c|c|c|}
\hline & Mean (SD) / \% & 1 & 2 & 3 & 4 & 5 & 6 & 7 & 8 & 9 & 10 & 11 & 12 & 13 & 14 & 15 & 16 & 17 \\
\hline 1 & $40.42(12.48)$ & - & .14 & -.11 & $-.19 * *$ & -.03 & $.23 * *$ & .04 & $.41 * * *$ & $.19 * *$ & .03 & $.21 * *$ & $.38 * * *$ & -.13 & $.28 * * *$ & $.30 * * *$ & .01 & $.36 * * *$ \\
\hline 2 & $89.8 \%$ female & & - & .12 & -.05 & -.01 & -.09 & .10 & -.10 & $.23 * *$ & $.24 * *$ & .09 & .04 & .01 & $-.16 * *$ & -.06 & .06 & -.01 \\
\hline 3 & $71.1 \%$ yes & & & - & $.33 * * *$ & .08 & -.14 & $.29 * * *$ & $-.14 *$ & .12 & $.24 * *$ & -.12 & $-.18 * *$ & $.16^{*}$ & -.01 & -.10 & .05 & $-.17 *$ \\
\hline 4 & $8.96(12.35)$ & & & & - & .06 & $-.24 * *$ & .11 & -.10 & $.36 * * *$ & $.35 * * *$ & -.13 & -.12 & .11 & -.07 & $-.15 *$ & .07 & $-.29 * * *$ \\
\hline 5 & $81.2 \%$ unexpected & & & & & - & $.26 * * *$ & $.16^{*}$ & -.02 & -.10 & $-.16^{*}$ & .12 & -.01 & -.07 & .06 & .10 & $-.17 *$ & .11 \\
\hline 6 & $56 \%$ no & & & & & & - & .09 & $.19 * *$ & -.07 & $-.17^{*}$ & $.27 * * *$ & $.21 * *$ & -.06 & $.22 * *$ & $.29 * * *$ & -.11 & $.30 * * *$ \\
\hline 7 & $33.09(28.07)$ & & & & & & & - & .05 & .13 & .09 & -.01 & -.01 & .10 & -.03 & -.06 & -.01 & -.04 \\
\hline 8 & $68.62(18.82)$ & & & & & & & & - & -.07 & $-.15^{*}$ & $.28 * * *$ & $.43 * * *$ & -.11 & $.36^{* *}$ & $.45^{* * *}$ & $-.20 * *$ & $.48 * * *$ \\
\hline 9 & $1.66(0.85)$ & & & & & & & & & - & $.60 * * *$ & .05 & .01 & .09 & -.09 & -.02 & $.16^{*}$ & -.09 \\
\hline 10 & $0.72(0.83)$ & & & & & & & & & & - & -.12 & -.05 & $.16^{*}$ & -.14 & $-.15^{*}$ & $.23 * *$ & $-.22 * *$ \\
\hline 11 & $3.10(1.10)$ & & & & & & & & & & & - & $.43 * * *$ & $-.32 * * *$ & $.27 * *$ & $.61 * * *$ & $-.15^{*}$ & $.58 * * *$ \\
\hline 12 & $3.50(0.86)$ & & & & & & & & & & & & - & $-.32 * * *$ & $.37 * *$ & $.62 * * *$ & $-.21 * *$ & $.60 * * *$ \\
\hline 13 & $1.86(0.78)$ & & & & & & & & & & & & & - & .01 & $-.36 * * *$ & $.39 * * *$ & $-.29 * * *$ \\
\hline 14 & $3.04(0.99)$ & & & & & & & & & & & & & & - & $.38 * * *$ & -.10 & $.39 * * *$ \\
\hline 15 & $3.20(1.09)$ & & & & & & & & & & & & & & & - & $-.40 * * *$ & $.78^{* * *}$ \\
\hline 16 & $2.19(0.93)$ & & & & & & & & & & & & & & & & - & $-.43 * * *$ \\
\hline 17 & $3.14(1.13)$ & & & & & & & & & & & & & & & & & - \\
\hline
\end{tabular}

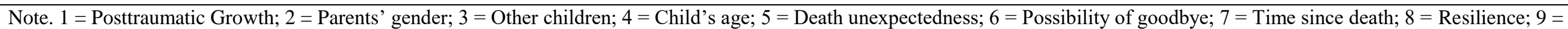

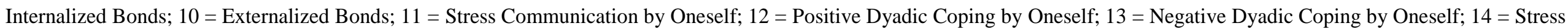
Communication by Partner; $15=$ Positive Dyadic Coping by Partner; $16=$ Negative Dyadic Coping by Partner; $17=$ Joint Dyadic Coping.

$* p<.05 ; * * p<.01 ; * * * p<.001$ 
Table 2. Summary of hierarchical regression analysis for variables predicting posttraumatic growth

\begin{tabular}{|c|c|c|c|c|c|c|c|c|c|}
\hline \multirow[t]{2}{*}{ Variable } & \multicolumn{3}{|c|}{ Model 1} & \multicolumn{3}{|c|}{ Model 2} & \multicolumn{3}{|c|}{ Model 3} \\
\hline & $B$ & $S E B$ & $\beta$ & $B$ & $S E B$ & $\beta$ & $B$ & $S E B$ & $\beta$ \\
\hline Parents' gender & 7.44 & 3.01 & $.18^{*}$ & 5.39 & 2.75 & .13 & 5.92 & 2.75 & $.14^{*}$ \\
\hline Other children & -1.89 & 2.09 & -.07 & -0.16 & 1.89 & -.01 & 0.03 & 1.89 & .001 \\
\hline Child's age & -0.01 & 0.01 & -.10 & -0.02 & 0.01 & $-.19 *$ & -0.02 & 0.01 & $-.18 *$ \\
\hline Death unexpectedness & -3.02 & 2.35 & -.09 & -1.15 & 2.11 & -.04 & -0.65 & 2.10 & -.02 \\
\hline Possibility of goodbye & 5.80 & 1.88 & $.23^{*}$ & 3.70 & 1.69 & $.15^{*}$ & 3.18 & 1.70 & .13 \\
\hline Time since death & 0.02 & 0.03 & .04 & -0.01 & 0.03 & -.02 & 0.00 & 0.03 & .000 \\
\hline Resilience & & & & 0.27 & 0.04 & $.40 * * *$ & 0.21 & 0.05 & $.32 * * *$ \\
\hline Internalized Bonds & & & & 4.21 & 1.16 & $.29 * * *$ & 4.26 & 1.15 & $.30 * * *$ \\
\hline Externalized Bonds & & & & 0.03 & 1.23 & .002 & -0.24 & 1.22 & -.02 \\
\hline $\mathrm{SCO}$ & & & & & & & -1.32 & 0.91 & -.12 \\
\hline PDCO & & & & & & & 1.82 & 1.20 & .12 \\
\hline $\mathrm{NDCO}$ & & & & & & & -2.13 & 1.15 & -.14 \\
\hline SCP & & & & & & & 1.81 & 0.88 & $.14 *$ \\
\hline PDCP & & & & & & & 0.48 & 1.13 & .04 \\
\hline NDP & & & & & & & 1.71 & 0.96 & .13 \\
\hline$\Delta R^{2}$ & & .103 & & & .207 & & & .057 & \\
\hline$F$ for $\Delta R^{2}$ & & $3.56 * *$ & & & $18.23 *$ & & & $2.62 *$ & \\
\hline
\end{tabular}

Note: $\mathrm{SCO}=$ Stress Communication by Oneself; PDCO = Positive Dyadic Coping by Oneself; NDCO = Negative Dyadic Coping by Oneself; SCP = Stress Communication by Partner; PDCP = Positive Dyadic Coping by Partner; NDCP = Negative Dyadic Coping by Partner

$* p<.05 ; * * p<.01 ; * * * p<.001$ 